\title{
Wavy Rib
}

National Cancer Institute

\section{Source}

National Cancer Institute. Wavy Rib. NCI Thesaurus. Code C124592.

A physical quality in which the rib is undulating. 